\title{
Tasty or Toxic: Evaluating the Effects of Common Food Additives on Daphnia Magna
}

\author{
Aditya Mahna, Ayush Mahna \\ Troy High School, 2200 Dorothy Ln, Fullerton, CA 92831, USA; adimahna@gmail.com
}

\begin{abstract}
The adverse effects of three of the most used food additives, MSG, food color, and sodium nitrite, were investigated in this research. The hypothesis was that these food additives would have harmful effects on Daphnia magna. Treatment groups called control, MSG, sodium nitrite, and food color each were tested in four different concentrations. Five Daphnia magna that were less than 24-hours-old were tested per group and were observed after thirty minutes and after overnight incubation with each of the additives. Heart rate and mobility were measured to determine the acute toxicity of the test compounds. In MSG, the heart rate decreased significantly in all concentrations after overnight incubation. All Daphnia numbers were deceased within thirty minutes in sodium nitrite in both $1 \%$ and $2 \%$ concentrations, showing that sodium nitrite is toxic. However, cardiac effects did not cause this because heart rate in lower concentrations of $0.1 \%$ and $0.01 \%$ did not change significantly from control. With food dye, the heart rate decreased significantly in concentrations as low as $0.1 \%$ after overnight incubation. Finally, we can conclude that these food additives have a toxic effect on Daphnia magna and the severity of these effects is based on the time of exposure and quantity of food additives consumed.

KEYWORDS: Toxicology; Food Additives; Daphnia magna; Monosodium Glutamate; Food Color; Sodium Nitrite.
\end{abstract}

\section{Introduction}

Foods have evolved and changed tremendously in the past decade. Food additives are now applied to many different food types. Food additives are substances used to preserve the food or enhance its flavor or appearance. Recent studies have shown that these substances may have negative effects on the human body. ${ }^{1,2}$ To test this, we decided to do experiments with the three most common food additives: MSG, food color, and sodium nitrite. MSG, or monosodium glutamate, is the sodium salt of glutamic acid, one of the most abundant naturally occurring, non-essential amino acids. It is used to intensify and enhance the flavor of food. ${ }^{3}$ Studies have shown that it can cause headaches, asthma, and even brain damage. ${ }^{4-6}$ Food coloring is made in a lab with chemicals derived from petroleum, a crude oil product, which is also used in gasoline, diesel fuel, asphalt, and tar. ${ }^{7}$ Food color is used to improve the appearance of foods and is often used in advertisements and fast-food restaurants. The excessive use of food color has been shown to reduce the attention span and cause ADHD and other behavioral problems in children.8,9 Sodium nitrite is often found in processed meats as a preservative to prevent bacteria growth. ${ }^{10}$ Studies have shown that over-consumption of sodium nitrite can cause kidney damage and low bloodpressure. ${ }^{11,12}$ We tested each food additive on Daphnia magna. D. magna is a tiny, semi-transparent freshwater crustacean with long antennae and prominent eyes. D. magna was used because they are a perfect model system, easy to handle, and are an ideal system for studying multiple stressors. They are good test subjects because of their transparent bodies, early reproduction rates, short lifespans, and gender change response system. We hypothesized that these food additives would have harmful effects on the D. magna.

\section{Results and Discussion}

To observe the short'term effects of food additives, D. magna were incubated with each of the test compounds for 30 minutes at four different concentrations: $2 \%, 1 \%, 0.1 \%$, and $0.01 \%$. These concentrations represent the range of relevant concentrations of food additives that can be found in the human body system. Five D. magna were included in each group. After 30 minutes the heart rates were measured. The average heart rate for the control group was 193 beats per minutes (bpm). No significant effect was observed in any groups for any concentration tested after 30 minutes of incubation. However, all of D. magna died within 30 minutes of incubation in $2 \%$ and $1 \%$ sodium nitrite and all D. magna died in the $2 \%$ food color group (Table 1 and Figure 1). At least one D. magna died in each of the concentrations tested in the food additive groups. No D. magna died in the control group (Table 3 and Figure 2). The deceased D. magna were not included in heart rate analysis.

To observe the effects of the food additives after longer incubation, D. magna were left overnight (12 hours) with food additives. The heart rates were measured the following day. After overnight incubation, the average heart rate for the control group was $173 \mathrm{bpm}$. The MSG and sodium nitrite groups displayed a dose-dependent increase in death of $D$. magna, indicating increased toxicity of these food additives with increased dosage (Table 3 and Figure 2). There was a significant reduction in heart rate in D. magna in the presence of MSG and food dye (Table 2 and Figure 1). Interestingly, $0.1 \%$ and $0.01 \%$ sodium nitrite did not display a significant 
change in heart rate compared to the control. However, all $D$. magna in $2 \%$ and $1 \%$ sodium nitrite died within 30 minutes. This indicates that sodium nitrite is highly toxic at higher concentrations but not due to cardiac toxicity.

Table 1: Heart rate in D. magna after 30-minute incubation with various concentrations of food additives. The black filled cells indicate the D. magna that died in the presence of food additives.

\begin{tabular}{|c|c|c|c|c|c|c|c|c|c|}
\hline & \multicolumn{8}{|c|}{ Heart Rate (30 min) } & \multirow[b]{3}{*}{$\begin{array}{c}\text { P Value } \\
\text { (Student's } \\
\text { t-Test) }\end{array}$} \\
\hline & & & & D. $r$ & agna i & ividu & & & \\
\hline & Concentration & D1 & D2 & D3 & D4 & D5 & Average & STDEV & \\
\hline Control & N/A & 200 & 196 & 192 & 194 & 184 & 193.2 & 5.9 & N/A \\
\hline \multirow{4}{*}{ MSG } & $2 \%$ & 184 & 196 & & 200 & 164 & 186 & 16.2 & 0.32 \\
\hline & $1 \%$ & 200 & 192 & 204 & & 200 & 199 & 5.0 & 0.30 \\
\hline & $0.1 \%$ & 204 & 188 & 208 & & 208 & 202 & 9.5 & 0.29 \\
\hline & $0.01 \%$ & 168 & 168 & 172 & 168 & & 169 & 2.0 & 0.00 \\
\hline \multirow{4}{*}{ Sodium Nitrite } & $2 \%$ & & & & & & & N/A & N/A \\
\hline & $1 \%$ & & & & & & & $N / A$ & N/A \\
\hline & $0.1 \%$ & 188 & 196 & & 168 & 184 & 184 & 11.8 & 0.22 \\
\hline & $0.01 \%$ & 184 & 188 & & 180 & 188 & 185 & 3.8 & 0.16 \\
\hline \multirow{4}{*}{ Food Dye } & $2 \%$ & & & & & & & N/A & N/A \\
\hline & $1 \%$ & 196 & & 200 & 176 & 192 & 191 & 10.5 & 0.82 \\
\hline & $0.1 \%$ & 196 & 180 & 172 & 196 & & 186 & 12.0 & 0.16 \\
\hline & $0.01 \%$ & 200 & & 184 & 212 & 224 & 205 & 17.1 & 0.33 \\
\hline
\end{tabular}

Table 2: Daphnia magna heart rate after 12-hour incubation with various concentration of food additives. The black filled cells indicate the D. magna died in the presence of food additives.

\begin{tabular}{|c|c|c|c|c|c|c|c|c|c|}
\hline \multirow[b]{2}{*}{ Name } & \multicolumn{9}{|c|}{ Heart Rate (Overnight) } \\
\hline & Concentration & D1 & D2 & D3 & $\mathrm{D} 4$ & D5 & Average & Standard Dev & $\begin{array}{c}\text { P Value } \\
\text { (Student's } \\
\text { t-Test) }\end{array}$ \\
\hline Control & $\mathrm{N} / \mathrm{A}$ & 168 & 184 & 176 & 188 & 152 & 174 & 14.3 & N/A \\
\hline \multirow{4}{*}{ MSG } & $2 \%$ & & 128 & 136 & & & 132 & 5.7 & N/A \\
\hline & $1 \%$ & 120 & 128 & & & 116 & 121 & 6.1 & 0.015 \\
\hline & $0.1 \%$ & 116 & 128 & & & 124 & 123 & 6.1 & 0.035 \\
\hline & $0.01 \%$ & 136 & 132 & 128 & & 156 & 138 & 12.4 & 0.087 \\
\hline \multirow{4}{*}{ Sodium Nitrite } & $2 \%$ & & & & & & N/A & N/A & N/A \\
\hline & $1 \%$ & & & & & & N/A & N/A & N/A \\
\hline & $0.1 \%$ & 176 & & & 160 & & 168 & 11.3 & 0.677 \\
\hline & $0.01 \%$ & 160 & 156 & & & 152 & 156 & 4.0 & 0.286 \\
\hline \multirow{4}{*}{ Food Dye } & $2 \%$ & & & & & & N/A & N/A & $\mathrm{N} / \mathrm{A}$ \\
\hline & $1 \%$ & 92 & 112 & 96 & 76 & 112 & 98 & 15.1 & 0.003 \\
\hline & $0.1 \%$ & 116 & 140 & 144 & 96 & & 124 & 22.4 & 0.024 \\
\hline & $0.01 \%$ & 180 & 164 & 156 & 120 & 144 & 153 & 22.5 & 0.189 \\
\hline
\end{tabular}

Table 2: Daphnia magna heart rate after 12-hour incubation with various concentration of food additives. The black filled cells indicate the D. magna died in the presence of food additives.

\begin{tabular}{|c|c|c|c|}
\hline Name & Concentration & \multicolumn{2}{|c|}{ \# of Mobile $D$. magna } \\
\hline \multirow{3}{*}{ Control } & & 30 min & Overnight \\
\hline \multirow{3}{*}{ MSG } & N/A & 5 & 5 \\
\hline \multirow{3}{*}{ Sodium Nitrite } & $2 \%$ & 4 & 2 \\
\cline { 2 - 4 } & $1 \%$ & 4 & 3 \\
\cline { 2 - 4 } & $0.1 \%$ & 5 & 3 \\
\cline { 2 - 4 } & $0.01 \%$ & 5 & 4 \\
\cline { 2 - 4 } & $2 \%$ & 0 & 0 \\
\cline { 2 - 4 } & $1 \%$ & 0 & 2 \\
\hline \multirow{3}{*}{ Food Dye } & $0.1 \%$ & 5 & 3 \\
\cline { 2 - 4 } & $0.01 \%$ & 0 & 0 \\
\cline { 2 - 4 } & $2 \%$ & 5 & 4 \\
\cline { 2 - 4 } & $1 \%$ & 5 & 5 \\
\hline
\end{tabular}

\section{- Discussion}

These results show that food additives have harmful effects on the health of Daphnia magna. Food dye had significant cardiovascular effects corresponding to its concentration. MSG slowed the heart rate of D. magna while sodium nitrite did not display any significant cardiovascular effects. However, sodium nitrite was shown to be extremely harmful in high concentrations as none of the D. magna were alive in trations above $0.1 \%$ after 30 minutes of incubation. MSG and food dye impacted mobility of Daphnia magna in a dose dependent manner. The dose dependent effects were highly evident after overnight incubation. This also shows that the

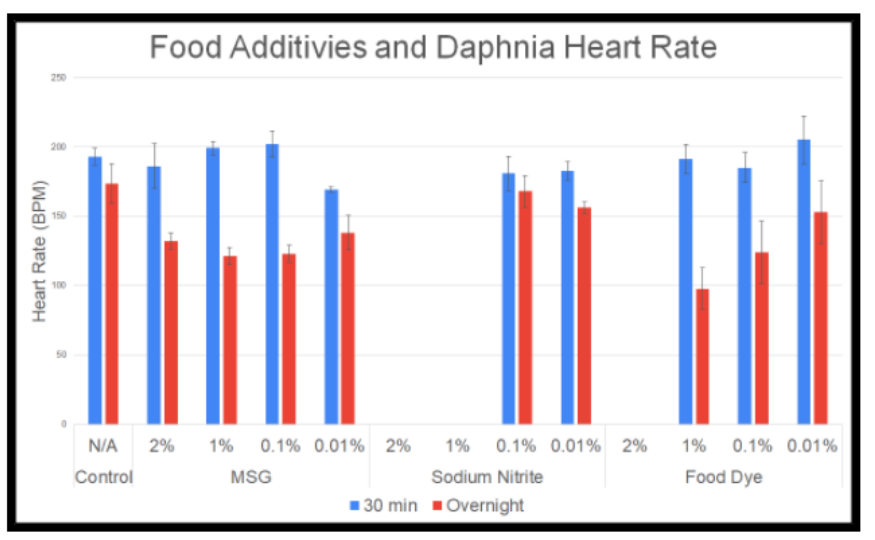

Figure 1: The effects of various food additives on Daphnia magna heart rate.

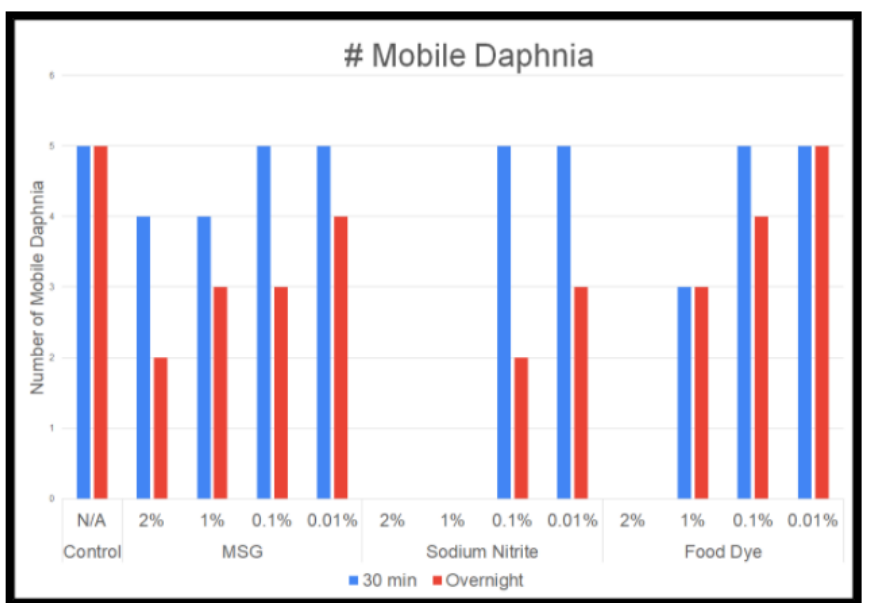

Figure 2: The effect of various food additives on the mobility of Daphnia magna.

overnight incubation had a much larger influence on D. magna than the 30-minute incubation. Overall, these results indicate that food additives have toxic effects on Daphnia magna and these effects are dependent on the concentration and time of exposure.

\section{- Conclusion}

Based on these results obtained in this study, we can conclude that these food additives have a toxic effect on Daphnia magna, and the severity of these effects are based on the time of exposure to these additives and the concentrations at which the additives are used. Using these results, further studies should be performed on humans using the hypothesis derived from this study. Although humans should portray the effects found in this study, they may not because of the variations between the physical and genetic makeup of the two species.

\section{Methods}

To determine the health effects of food additives, the Daphnia magna were tested in four groups (control, MSG, sodium nitrite, and Quinoline yellow food color). Each food 
additive was tested in four different concentrations $(0.01 \%$, $0.1 \%, 1 \%$, and $2 \%)$. D. magna heart rate and mobility were measured as an indicator of toxicity. Five one-day-old $D$. magna were tested per group. The control group was left in spring water without any additives. The measurements for heart rate and mobility were performed after 30 minutes and overnight incubation with each food additive. The heart rate was measured by looking at D. magna through the microscope and counting how many beats occurred in 15 seconds. The results were multiplied by 4 to get the beats per minute (bpm). Lastly, to determine the results of mobility, the test container with $D$. magna was shaken and then observed to see if any $D$. magna moved within fifteen seconds. The results were entered into Excel and graphs were created. The results were analyzed by calculating the average heart bpm and standard deviation. The number of mobile D. magna was recorded by counting. The Excel data analysis tool ANOVA was used to determine if there was a significant effect due to any food additive. Once ANOVA determined that there was a significant effect due to food additive, a two-tailed, paired student t-test was performed to determine the significance of effects at each concentration of food additive in comparison to the control group. A p-value less than 0.05 was considered significant.

\section{- Acknowledgement}

We thank Dr. Suman Verma for guiding and advising us throughout this project and Carolina Biological for providing us with the Daphnia magna as an ideal model system. Special thanks to Professor Bruce Blumberg from University of California, Irvine for advising and guiding us along this project and for giving us the opportunity to conduct our experiment in his lab. This research won first prize in the Orange County Science \& Engineering Fair in the Toxicology Division and continued on to the California State Science Fair.

\section{References}

1. EFSA ANS Panel; Mortensen, A.; Aguilar, F.; Crebelli, R.; Di Domenico, A; Dusmund, B.; Frutos, M.J.; Galtier, P.; Gott, D.; Gundert-Remy, U.; et al. Re-evaulation of sodium nitrate (E 251) and potassium nitrate (E 252) as food additives. EFSA J. 2017, 15(6), 4787. DOI: 10.2903/ j.efsa.2017.4787.

2. Lee, D.; Swan, C. K.; Suskind, D.; Wahbeh, G.; Vanamala, J.; Baldassano, R. N.; Leonard, M. B.; Lampe, J. W. Children with Crohn's Disease Frequently Consume Select Food Additives. Dig. Dis. Sci. 2018, 63(10), 27222728. DOI: $10.1007 / \mathrm{s} 10620-018-5145-x$.

3. Onuma, T.; Maruyama, H.; Sakai, N. Enhancement of Saltiness Perception by Monosodium Glutamate Taste and Soy Sauce Odor: A Near-Infrared Spectroscopy Study. Chem. Senses 2018, 43(3), 151-167.

DOI: $10.1093 /$ chemse/bjx084.

4. Shimada, A.; Cairns, B. E.; Vad, N.; Ulriksen, K.; Pedersen, A. M. L.; Svensson, P.; Baad-Hansen, L. Headache and mechanical sensitization of human pericranial muscles after repeated intake of monosodium glutamate (MSG). J. Headache Pain 2013, 14(1), 2. DOI:10.1186/1129-2377-14-2

5. Sharma, A.; Prasongwattana, V.; Cha'on, U.; Selmi, C.; Hipkaeo, W.; Boonnate, P.; Pethlert, S.; Titipungul, T.; Intarawichian, P.; Waraasawapati, S.; et al.
Monosodium Glutamate (MSG) Consumption is Associated with Urolithiasis and Urinary Tract Obstruction in Rats. PloS One 2013, 8(9), e75546. DOI: 10.1371/ journal.pone.0075546.

6. Zanfirescu, A.; Ungurianu, A.; Tsatsakis, A. M.; Nitulescu, G. M.; Kouretas, D.; Veskoukis, A.; Tsoukalas, D.; Engin, A. B.; Aschner, M.; Margină, D. A Review of the Alleged Health Hazards of Monosodium Glutamate. Compr. Rev. Food Sci. Food Saf. 2019, 18(4), 1111-1134. DOI: 10.1111/1541-4337.12448.

7. Bell, B. Food Dyes: Harmless or Harmful? https://www.healthline.com/nutrition/food-dyes\#: : text $=$ What $\% 20$ Are\%20Food\%20Dyes\%3F,dyes\%20are\% 20made\%20from\%20petroleum. (Jan. 7, 2017)

8. Cheeseman, M. A. Artificial Food Color Additives and Child Behavior. Environ. Health Perspect. 2012, 120(1), a15-a16. DOI: 10.1289/ehp.1104409.

9. Arnold, L E.; Lofthouse, N.; Hurt, E. Artificial food colors and attention-deficit/hyperactivity symptoms: conclusions to dye for. Neurotherapeutics 2012, 9(3), 599609. DOI: $10.1007 / \mathrm{s} 13311-012-0133-x$.

10. EFSA ANS Panel; Mortensen, A.; Aguilar, F.; Crebelli, R.; Di Domenico, A; Dusmund, B.; Frutos, M. J.; Galtier, P.; Gott, D.; Gundert-Remy, U.; et al. Re-evaluation of potassium nitrite (E 249) and sodium nitrite (E 250) as food additives. EFSA J. 2017, 15(6), 4786. DOI: 10.2903/ j.efsa.2017.4786.

11. Feelisch, M.; Akaike, T.; Griffiths, K.; Ida, T.; Prysyazhna, O.; Goodwin, J. J.; Gollop, N. D.; Fernandez, B. O.; Minnion, M.; Cortese-Krott, M.; et al. Longlasting blood pressure lowering effects of nitrite are NOindependent and mediated by hydrogen peroxide, persulfides, and oxidation of protein kinase G1a redox signalling. Cardiovasc. Res. 2020, 116(1), 51-62.

DOI: $10.1093 / \mathrm{cvr} / \mathrm{cvz} 202$

12. Rosenbaek, J. B.; Pedersen, E. B.; Bech, J. N. The effect of sodium nitrite infusion on renal function, brachial and central blood pressure during enzyme inhibition by allopurinol, enalapril or acetazolamide in healthy subjects: a randomized, double-blinded, placebocontrolled, crossover study. BMC Nephrol. 2018, 19(1), 244. DOI: $10.1186 / s 12882-018-1035-x$.

\section{Author}

Aditya Mahna is a student attending Troy High School who loves doing research and exploring new topics. He hopes to major in biology in college and continue investigating the effects of food and dietary substances on human health.

Ayush Mahna is a young scientist who enjoys the field of biology and exploring the effects of common diets on human health. He wishes to major in computer science in college to unravel biological mysteries through data analysis. 